\title{
Science Goals and Mission Objectives for the Future Exploration of Ice Giants Systems: a Horizon 2061 Perspective
}

\section{Part II: From Measurement Requirements to Mission Scenarios}

\author{
A White Paper prepared for the \\ NAS 2023-2032 Decadal Survey for Planetary Science and Astrobiology \\ August 15th, 2020
}

Contact author: Michel Blanc1,2, Cell +33659101290 , IRAP, CNRS-UPS-CNES, Toulouse, France - email : michel.blanc@irap.omp.eu

Co-authors: Kathleen Mandt3, Olivier Mousis2, Nicolas André1, David H. Atkinson4, Sushil Atreya18, Tibor Balint4, Scott Bolton17, Alexis Bouquet2, Sébastien Charnoz4, Kate Craft3, Magali Deleuil2, Leigh Fletcher20, Léa Griton1, Ravit Helled5, Mark Hofstadter4, Sean Hsu13; Ricardo Hues06, Xianzhe Jia18, Laurent Lamy7, Jonathan Lunine8, Marius Millot19, Glenn Orton4, Frank Postberg14, Thomas Ronnet,, Kunio M. Sayanagi15, Juergen Schmidt10, Krista Soderlund11, Linda Spilker4, Tom Spilker16, Diego Turrini12, Elizabeth Turtle3, Pierre Vernazza2, J. Hunter Waite17

Affiliations: IIRAP, CNRS-Université Toulouse III, France; 2LAM, CNRS-AixMarseille Université, Marseille, France ; 3JHU-APL, Laurel, MD, USA ; 4Jet Propulsion Laboratory, California Institute of Technology, Pasadena, CA, USA; 4Institut de Physique du Globe de Paris; 5 University of Zurich, Switzerland ; 6 Universidad del País Vasco (UPV/EHU); 7LESIA, CNRS-Observatoire de Paris, Meudon, France ; 8Cornell University, Ithaca, NY, USA ; gLund University, Sweden; 10 University of Oulu, Finland; 11Institute for Geophysics, Jackson School of Geosciences, University of Texas at Austin, Austin, TX, USA ; 12 Institute for Space Astrophysics and Planetology INAFIAPS, Italy; ${ }_{13}$ University of Colorado Boulder, USA; 14 Freie Universität Berlin, Germany; 15 Hampton University, Hampton, VA, USA; 16 Independent consultant; 17SwRI, San Antonio, TX, USA; 18 University of Michigan, Ann Arbor, USA; 19 Lawrence Livermore National Laboratory, USA; 20 University of Leicester, UK.

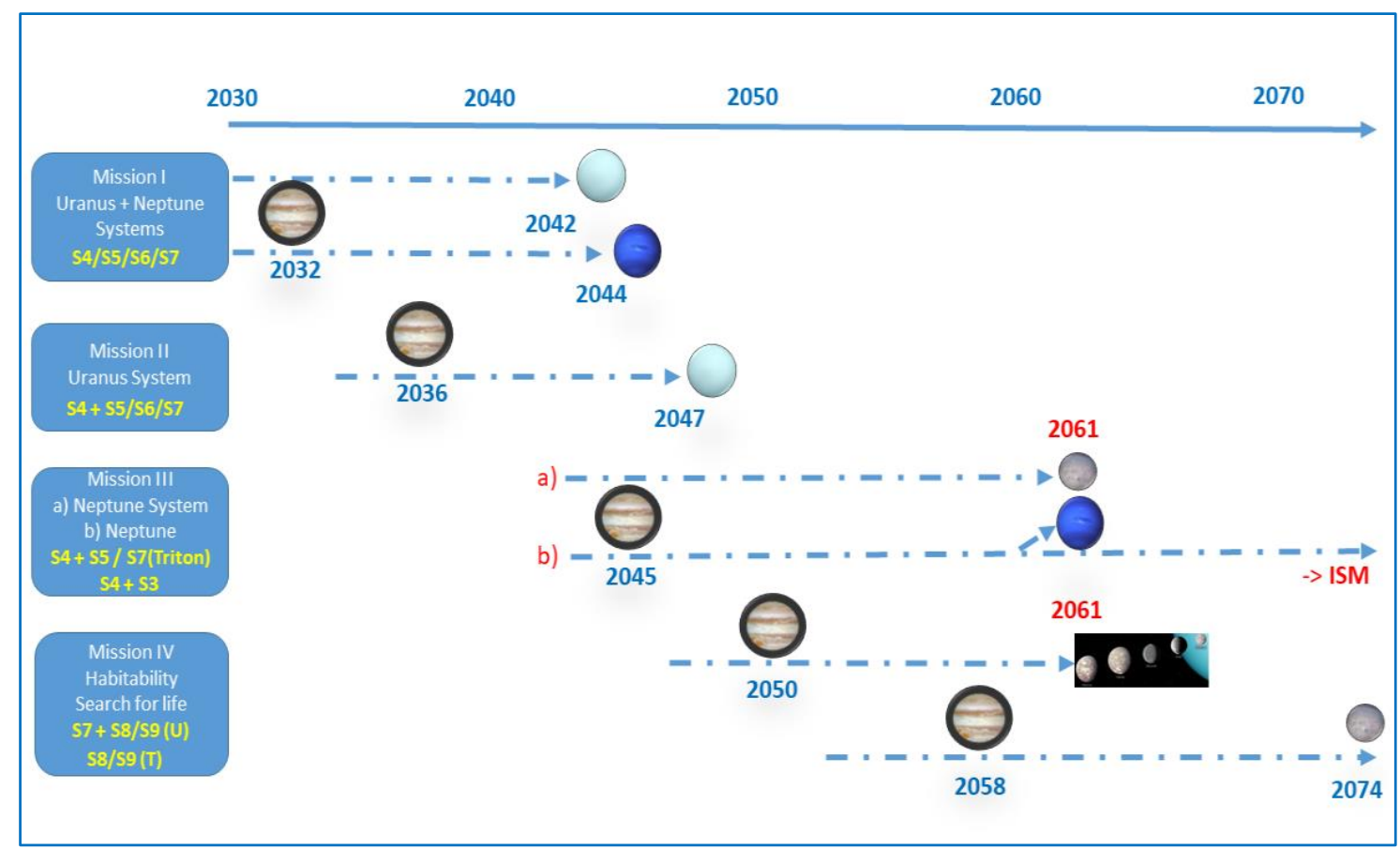

ICE GIANTS MISSIONS NOTIONAL ROADMAP 


\section{Introduction}

Planetary science has experienced in the last two decades the emergence of a new unifying paradigm: the concept of "planetary system", which links together the solar system, giant planet systems and extrasolar planetary systems. Among them, primary planetary systems orbit a star while secondary planetary systems, including the four Giant Planets systems, have a planet as their central object.

The "Planetary Exploration, Horizon 2061" foresight exercise (http://horizon2061.cnrs.fr/) has proposed six key science questions, Q1 to Q6, for the comparative study of planetary systems:

Q1- How well do we understand the diversity of planetary system objects?

Q2- How well do we understand the diversity of planetary system architectures?

Q3- What are their origins and formation scenarios?

Q4- How do they work?

Q5- Do they host potential habitats?

Q6- Where and how to search for life?

The legacy of 20th century solar system exploration, despite its wealth of discoveries, leaves a huge gap in their exploration: the two ice giant systems have been visited only once by the fly-by of the same probe, Voyager 2, in 1986 and 1989. In part I of this White Paper, based on a comprehensive article in which more details can be found [1], we showed how the six "key questions" can be addressed in depth at ice giant systems and identified measurement requirements for missions to these systems.

In part II, this current White Paper, we now describe a sequence of missions to the Ice Giants that can perform the key measurement requirements identified in part I.

\section{Reference flight science sequences}

We suggest that the measurements required to address the six key science objectives of Horizon 2061 will be performed by ice giants missions using a succession of typical flight science sequences which are currently used in the design of missions to giant planet systems. These configurations can be classified in terms of eleven notional flight science sequences, S1 to S11. For each sequence, table I gives its main science applications and examples of space missions using it. Let us briefly comment it.

S1 "Earth-based Observations" corresponds to the observations of planetary objects performed from the ground or from Earth-Moon system orbits. Building on the HST legacy, the role of these remote observations should remain very important in the coming decades with the advent of JWST and giant space telescopes in space and with Extremely Large Telescopes on the ground, which will offer unprecedented spatial and spectral resolution.

S2 "Interplanetary cruise": in addition to the opportunities this mandatory phase of a planetary mission offers for heliospheric science, its approach phase to a planet can be used to study the coupling of the planet to the interplanetary medium, for instance by monitoring at the same time the variations in the planet's radio and auroral emissions. The long and continuous observation time offered by an orbiter en route to a giant planet could also be used for planetary seismology studies that would complement other approaches to the determination of the internal structure, given an instrument adapted to such a study is available on board [2] [3].

S3 "Planetary fly-by": a "simple" fly-by of an ice giant with state-of-the art instruments can nowadays provide an enormous science return compared to Voyager. Close (low altitude) fly-bys allowing measurements of the gravity and magnetic field along the arc of the fly-by, combined with imaging and passive microwave radiometry, can provide unique information on ice giants internal structure and atmospheric composition. 


\begin{tabular}{|c|c|c|c|}
\hline $\begin{array}{l}\text { FSS } \\
\#\end{array}$ & $\begin{array}{l}\text { Flight sequence } \\
\text { name }\end{array}$ & Main science addressed & Example(s) \\
\hline S1 & $\begin{array}{l}\text { Earth-based } \\
\text { Observations }\end{array}$ & $\begin{array}{l}\text { All disciplines but mainly } \\
\text { atmosphere, rings and satellites. } \\
\text { Access to multi-decadal temporal } \\
\text { variations }\end{array}$ & $\begin{array}{l}\text { HST, JWST, } \\
\text { EELT... }\end{array}$ \\
\hline S2 & $\begin{array}{l}\text { Interplanetary } \\
\text { cruise }\end{array}$ & $\begin{array}{l}\text { 2.e/4.c - Heliosphere, } \\
\text { Interplanetary medium effects on } \\
\text { magnetosphere }\end{array}$ & $\begin{array}{l}\text { Cassini@Jupiter\&S } \\
\text { aturn, } \\
\text { Juno@Jupiter }\end{array}$ \\
\hline S3 & Planetary fly-by & All disciplines & Voyager \\
\hline S4 & $\begin{array}{l}\text { Atmospheric } \\
\text { entry probe }\end{array}$ & $\begin{array}{l}1 \text { - Origins; } 3 . b-\text { Early dynamics of } \\
\text { the Solar System; } 2 . b \text { - interiors } \\
\text { and atmospheres }\end{array}$ & Galileo, Huygens \\
\hline S5 & $\begin{array}{l}\text { Synoptic orbital } \\
\text { survey of the } \\
\text { system }\end{array}$ & $\begin{array}{l}2 \& 3 \text { - Diversity of systems and } \\
\text { objects; } 4 \text { - How do these systems } \\
\text { work? }\end{array}$ & $\begin{array}{l}\text { Galileo, Cassini, } \\
\text { JUICE }\end{array}$ \\
\hline S6 & $\begin{array}{l}\text { Quasi-polar, low } \\
\text { periapse multiple- } \\
\text { orbit survey }\end{array}$ & $\begin{array}{l}1 \text { - origins; } 2 . b-\text { interiors and } \\
\text { atmospheres; } 3 . e \text { - diversity of } \\
\text { magnetospheres }\end{array}$ & $\begin{array}{l}\text { Juno, } \\
\text { Cassini proximal } \\
\text { orbits }\end{array}$ \\
\hline S7 & $\begin{array}{l}\text { Multiple } \\
\text { satellite(s) fly-bys }\end{array}$ & $\begin{array}{l}\text { 1- Origins; } 2 . c \text { - diversity of moons } \\
3 . d \text { - diversity of moon systems; }\end{array}$ & $\begin{array}{l}\text { Cassini, } \\
\text { Europa Clipper, } \\
\text { JUICE }\end{array}$ \\
\hline S8 & $\begin{array}{l}\text { Planetary moon } \\
\text { orbital survey }\end{array}$ & $\begin{array}{l}\text { 2.c- diversity of moons; } 5 \text { - moons } \\
\text { activity and habitability }\end{array}$ & JUICE \\
\hline S9 & $\begin{array}{l}\text { Planetary Moon } \\
\text { Lander }\end{array}$ & \multirow[t]{2}{*}{$\begin{array}{l}5 \text { - moons activity and habitability; } \\
6 \text { - search for life }\end{array}$} & $\begin{array}{l}\text { Huygens, Europa } \\
\text { lander }\end{array}$ \\
\hline S10 & $\begin{array}{l}\text { Planetary Moon } \\
\text { mobile element }\end{array}$ & & Dragonfly \\
\hline S11 & $\begin{array}{l}\text { Planetary Moon } \\
\text { sample return }\end{array}$ & $\begin{array}{l}5,6 \text { - Search for life; } \\
1 \text { Origins; } 2,3 \text { - diversity }\end{array}$ & $\begin{array}{l}\text { Apollo, MSR, } \\
\text { MMX-Phobos, } \\
\text { Caesar, future ice- } \\
\text { drilling missions }\end{array}$ \\
\hline
\end{tabular}

Table 1 - Flight science sequences (S1 to S11) used in the mission plans of missions to giant planets systems to design comprehensive tours of these systems.

S4 "Atmospheric entry probe" will provide unique information on a broad spectrum of science questions, mainly the origins of ice giants and their atmospheric composition, and help constrain the determination of their internal structure and heat flow, as has been described extensively in [4] [5].

S5 "Synoptic orbital survey of the system" is a series of orbits at low-to-medium inclinations, out of the equatorial plane, needed to provide a fair coverage of latitudes (for atmospheric studies), phase angles (for rings studies) and of the different magnetospheric regions. 
S6 "Quasi-polar, low periapse multiple-orbit survey", in contrast to S5, uses a very high inclination and a low periapse to fly very close to the planet and, over a series of successive orbits, provide a good coverage of longitudes to describe the main planetary fields (magnetic, gravity, plasma) and perform passive far IR and/or microwave sounding of its atmosphere, an approach used by the Juno mission [6]. S7 "Multiple moon fly-bys" allows one to characterize moons shapes, surface characteristics, exosphere, gravity and magnetic fields. This approach, extensively used by Cassini, Europa Clipper and JUICE, will be well suited for the study of the Uranian moons and can also prove effective for Triton [7].

S8, S9 and S10 correspond to sequences of increasing complexities and science returns for the study of a single planetary moon: first a series of polar orbits (S8) similar to the JUICE final sequence in orbit around Ganymede; then landing on the moon's surface to characterize a specific site, its surface and its coupling to the sub-surface and atmosphere/exosphere (S9); and finally a mobile element (S10).

S11: Finally, the ultimate way of studying the origin of a planetary moon and the astrobiological information it can contain is a sample return. We list it here for the sake of completeness but do not believe it can be implemented at the ice giants by 2061 .

2. Tentative science traceability matrix for the six key science questions. Based on the analysis of the six science questions presented in part I of this WP, we designed a synthesis table for the exploration of ice giants systems (Table 2). The lightblue boxes display the main measurements corresponding to each science question for the atmosphere and interior of the planet, its rings, moons and magnetosphere.

Then the four right-hand columns show how well the different possible destinations of missions to ice giant systems potentially address the six science questions. We have introduced in the "destination" columns one for combined missions to both giants and one for Triton, given its outstanding place in the family of ice giant moons.

Inspection of each horizontal line in Table 2 allows one to determine a preferred strategy to address each of the six science questions.

Q1- Diversity of objects: Uranus and Neptune are sufficiently different (see Table 1 in [1]) to require that both planets be visited with two suites of properly intercalibrated scientific instruments. As much as possible, one probe at each planet (S4) is needed, complemented preferentially by an S6 orbital mission or, minimally, by a fly-by (S3) providing measurements of internal planetary fields and passive microwave sounding of the atmosphere. It would be very relevant in terms of scientific added value to take advantage of another mission on its way to a farther destination, like TNO's, heliopause boundaries or the interstellar medium.

Q2- Diversity of systems architectures: characterizing in depth the different elements of the architecture of at least one of the ice giants systems is required. Uranus has the additional advantage that it hosts a system of endogenic regular moons. The Uranian and Neptunian rings systems and magnetosphere are qualitatively just as interesting and sufficiently different from their gas giant counterparts to deserve an in-depth exploration using an S5 flight sequence. Between the two systems, the larger coverage of the rings and magnetospheres parameter space at Uranus points to a slight preference for this destination.

Q3- Origin of Ice Giants systems: In-situ measurements of key elemental and isotopic abundances in at least one the ice giants, complemented by a low-periapse, high inclination orbiter (S6) "à la Juno" performing microwave remote sensing of the atmosphere [17] and constraining the internal structure from gravity and magnetic field measurements will revolutionize our understanding of the origins and evolution of ice giants. 


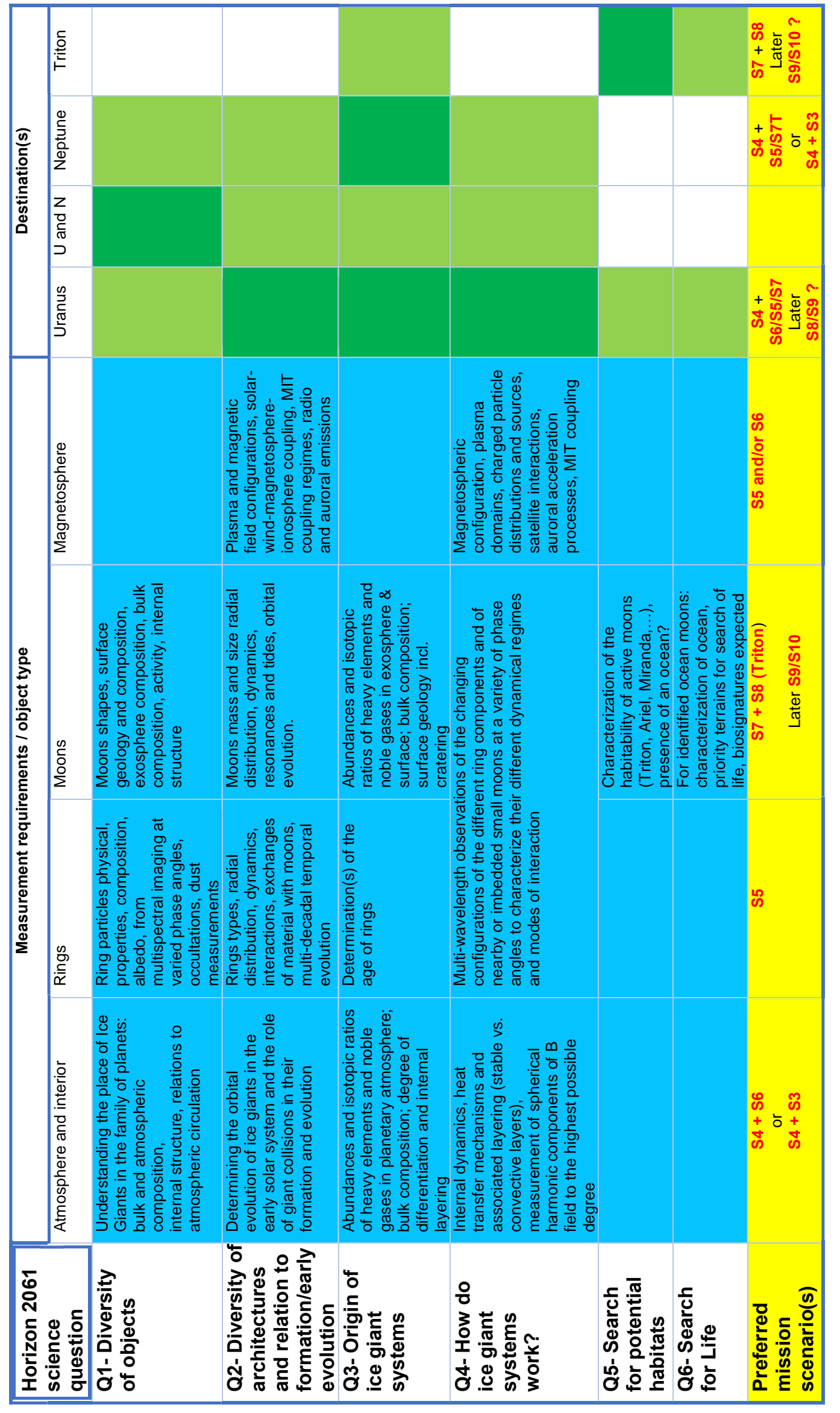

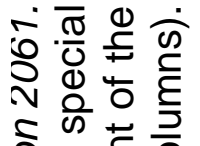

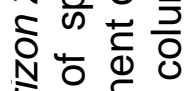
회웡응응 ธ。 क 으등 옹 \& 옹 음흑 ه. क 옹 도읃 웡 $\frac{}{0}$ 은 以 웡 के (1) 홍 इ 증 흥 등 Ð ర ธิ 乌 $=\frac{0}{0}$ 은 은 언 क क ष है도 하음 के ग ठ क $₫$ w

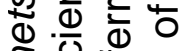
สิ 등 흘 듕 ชิ 1 응 के क्ष है 윤웡ㅇㅇ क ⿻上 을 을 은 ஸ 0 ह. 호웡 $\times>$ 총응 즌은 응 흐을 客 0 \& o $\times$ 촌 - 3 엉 응 응음 बิ 认 ง ㄷำ (1) 宁ष 
Complementary measurements of the bulk and surface composition of the most primitive moons and of their internal structure, surface geology and cratering history, preferentially at Uranus where the regular moons may have been co-generated with their host planet, will provide a similar progress in our understanding of the origin of these moons and of the system as a whole. On the other hand, Triton in itself bears a strong interest, as a captured TNO, to study the origin and formation scenario of the solar system as a whole.

Q4- How do Ice Giants systems work? We suggest to focus the study of the "workings" of ice giants systems on a few science themes: internal dynamics and their implications for heat transfer and the dynamo; atmospheric circulation; magnetospheric dynamics and its coupling to the upper atmosphere. Performing this study at both planets would be invaluable, particularly to understand the mystery of their very different external radiation flux and for a better characterization of solar system ice giants in view of their comparison to exoplanets of a similar class (see section 2.1 in [1] and [14]).

Q5- Search for potential habitats: based on our current knowledge, Triton is the best candidate for a habitable moon in the two systems. Characterizing its habitability should be one of the highlights of the first dedicated mission to the Neptune system. Following a scenario similar to JUICE at Ganymede, a mission to the Neptunian system should end up with multiple Triton flybys (S7) or even better a Triton orbiter after a first phase in Neptune orbit (preferably S6, then possibly S5).

Q6- Search for life: once the prerequisites for the search for life have been established and the best candidate habitable moon has been identified (likely Triton, or one of the active Uranian moons), a second mission dedicated to the search for life at the best candidate planet could be designed and launched before 2061 .

When read across all questions, Table 2 tells us that, in order to properly address all six questions at the ice giants, one dedicated mission to each of the systems will be needed. However, visiting only one of the two systems first will address many of these questions, and will be a huge step forward. Between the two systems, visiting Uranus first makes sense to address our first four questions: characterize ice giants in the family of planets, their systems in the family of planetary systems, understand their origin and formation scenarios, and study how they work. The corresponding preferred mission scenario is the combination of an atmospheric probe (S4) with an orbiter mission using flight sequences S6/S5/S7 in a t.b.d. order: a high-inclination sequence to probe the interior and atmosphere, a mid-inclination sequence to observe atmosphere, rings and magnetosphere, and multiple flybys of the regular moons.

On the other hand, the Neptune system is potentially a better choice to address the last two science questions, e.g. the habitability of ocean moons and the search for life there. Triton will naturally focus much attention in such a mission, whose preferred profile, after delivery of a probe (S4) could be a sequence S5/ S7@Triton, ending in orbit around Triton in a scenario similar to JUICE@Ganymede.

\section{From mission requirements to notional mission scenarios}

Studying the implementation of these scenarios in the Horizon 2061 time frame would require a detailed analysis of launch opportunities and enabling technologies which is far beyond the scope of this WP. We only use here the studies performed for the latest Decadal survey [7] and the ESA Ice Giants CDF study report [8].

With conventional launchers used for planetary missions (Ariane, Atlas...) any mission trajectory within the 2061 timeframe will have to include several planetary gravity assists, and a flyby of Jupiter will be mandatory. Uranus and Neptune do not align, so a single spacecraft cannot visit both planets [7], as was possible for the Voyager grand 
tour. For Uranus, the next possible transfers from Jupiter occur in 2036, 2050 and 2064, with a journey to destination in 11 years minimum in order to keep an arrival velocity compatible with an orbit insertion. The launch date from Earth is 2-5 years earlier, therefore in the ranges 2031-2034, 2045-2048 and 2059-2062 respectively. For Neptune, the next possible transfers from Jupiter occur in 2032, 2045 and 2058, with a minimum 16 years journey time to destination. To keep a reasonable mission duration, Earth launch dates could be 2 years earlier, e.g. 2030, 2043 and 2056. An atmospheric probe could also be delivered to Uranus or Neptune by a mission on its way to the interstellar medium, if compatible with its resources.

Finally, let us mention the interesting opportunity of a dual spacecraft dual-planet single launch in February 2031 studied in [7] and [8]. A SLS-IB heavy lift launch vehicle could send a composite of the Uranus and the Neptune orbiters directly to Jupiter together with a solar electric propulsion stage. The orbiters could carry an atmospheric probe. The two orbiters would separate during the transfer to Jupiter and perform independent Jupiter swing-by's in December 2032: a very close swing-by at a perijove altitude of $\sim 10,000 \mathrm{~km}$ for the Uranus orbiter, and a much higher one at $\sim 857,000 \mathrm{~km}$ for the Neptune orbiter, after which the two spacecraft would reach their targets in April 2042 and September 2044, respectively. This scenario provides an implementation of the scenario described in the ODINUS dual ice giants mission concept [2].

By allocating our preferred mission scenarios for each of the four possible destinations (bottom line of Table 2) to the different Jupiter fly-by windows to Uranus and Neptune just described, we built the sequence of mission opportunities, named Mission I to IV, illustrated from top to bottom in Figure 1. Mission I takes advantage of the dual Jupiter fly-by opportunity mentioned above to send two missions to the two ice giants simultaneously. If this opportunity cannot be met, one should plan to take advantage of the next two windows, one to Uranus with Jupiter fly-by in 2036 (mission II), one to Neptune with Jupiter fly-by in 2045 (mission IIIa), to fly a mission with the preferred profile to each of these planets. If only one of the two missions can be afforded, we suggest to fly mission II to Uranus and take the opportunity of a fly-by of Neptune by a different mission to the outer solar system, for instance a mission to the TNOs, the heliosphere boundaries and/or the local interstellar medium [12] [13], to deliver a probe into Neptune's atmosphere. This is what we identify as mission IIlb.

Finally, a follow-on mission dedicated to the search for life, either at one of the active moons of Uranus (mission IVa), or at Triton (mission IVb), could be designed and launched before 2061 once the best candidate for habitability has been identified by missions II and III. Mission IVa would fly by Jupiter in 2050, while mission IVb would fly by Jupiter in 2058: a very appealing window for an astrobiology mission to Triton.

\section{NASA-ESA collaboration opportunities}

Past studies by NASA and ESA (e.g. [7], [8]) have opened the way to international collaborations [15], showing that, from the technical point of view, a joint mission to an ice giant including an orbiter and an atmospheric probe could be developed, following a scenario similar to Cassini-Huygens. Dual spacecraft mission scenarios, with NASA and ESA flying jointly two orbiters to each of the ice giants, would also be technically feasible. Implementation of these collaboration opportunities will depend on the outcome of the current NAS Decadal Survey for Planetary Science and Astrobiology and, on the ESA side, of the Voyage 2050 planning exercise, to which three White Papers dealing with the exploration of Ice Giants systems have been submitted [9] [10] [11]. See also the follow-on publication of a science case for orbital science [16]. 


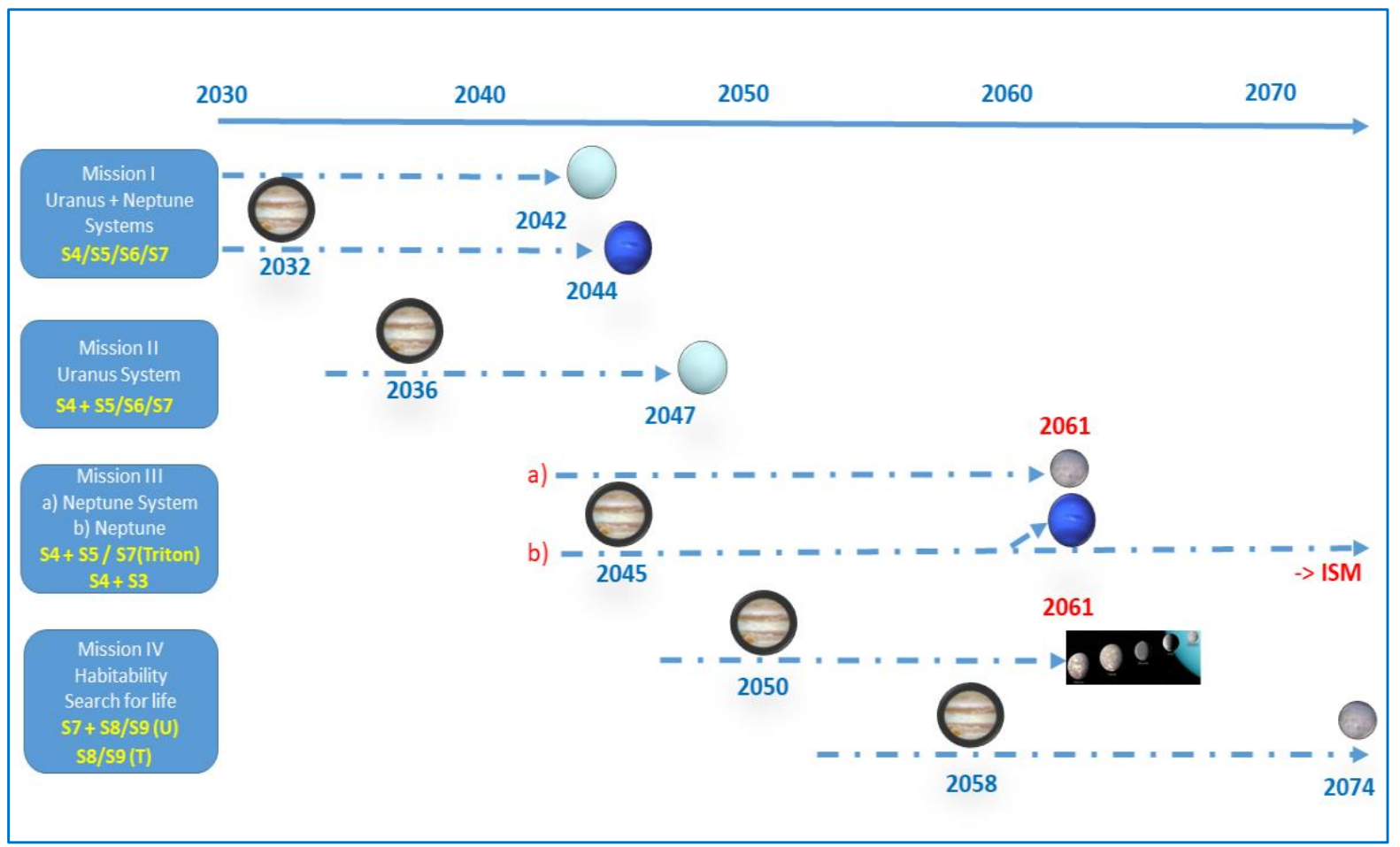

Figure 1: An illustration of the different mission opportunities offered by the fly-bys of Jupiter en route to ice giants up to 2061, together with the preferred flight sequences for each of these windows and destinations (see text).

Acknowledgements: KMS acknowledge support from NASA Grant NNX15AL56G. The French coauthors acknowledge support from CNES for Ice Giant Missions and CNRS/INSU programs of planetology and heliophysics. LL acknowledges the Hubble Space Telescope (Program GO/DD 14036) and the APIS service http://apis.obspm.fr, hosted by the Paris Astronomical Data Centre. Part of this work was performed by Lawrence Livermore National Laboratory under Contract DE-AC52-07NA27344.

References: [1] M. Blanc et al. Space Sci. Rev. submitted, 2020. [2] D. Turrini et al., Planet. Space Sci. 104, 93 (2014). [3] Gaulme P., B. Mosser, F.-X. Schmider, T. Guillot, in « Extraterrestrial seismology », edited by V. Tong and R. Garcia, Cambridge University Press, 189-202, 2015. [4] O. Mousis et al., The Astrophysical Journal 696, 1348 (2009). [5] K.E. Mandt, O. Mousis, and S. Treat. Monthly Notices of the Royal Astronomical Society 491, 488 (2020). [6] Bolton et al., Eds. The Juno Mission, Springer (2018). [7] Hosftadter et al. Planet. Space Sci. 177, 104680 (2019). [8] ESA Ice Giants CDF study report (2019). [9] Fletcher, L., Ice Giant Systems: Scientific Potential of Missions to Uranus and Neptune, White Paper submitted to ESA Voyage 2050 call (2019). [10] Guillot, T., Uranus and Neptune are key to understand planets with hydrogen atmospheres, White Paper submitted to ESA Voyage 2050 call (2019). [11] Mousis, O., In Situ Exploration of the Giant Planets, White Paper submitted to ESA Voyage 2050 call (2019). [12] McNutt et al., EPSC abstract (2019). [13]. Brandt et al., EPSC abstract (2019). [14] Deleuil et al. Space Sci. Rev., accepted, August 2020. [15] Simon et al., Space Sci Rev., 216:17 (2020). [16] Fletcher, L., Planet Space Sci. accepted 2020. [17] Atreya et al. SSRv 216:18, 2020. 